Antiretroviral-based HIV-1 prevention strategies - including antiretroviral treatment (ART) to reduce the infectiousness of HIV-1 infected persons and antiretroviral pre-exposure prophylaxis (PrEP) for uninfected persons to prevent HIV-1 acquisition - are promising new approaches for decreasing HIV-1 spread. The past two years have seen significant new advances in knowledge regarding ART and PrEP for HIV-1 prevention, including definitive demonstration in randomised trials that both ART and PrEP reduce HIV-1 risk and the development of normative guidance for prescribing these HIV-1 prevention strategies. Adherence is key to efficacy of antiretrovirals for HIV-1 prevention, both as ART and PrEP. There are numerous parallels in the challenges and opportunities ahead for ART and PrEP, including how to target to realise maximum population benefits, whether HIV-1 infected persons at earlier stages of infection would accept ART to reduce their risk for transmitting HIV-1 and highest-risk HIV-1 negative persons would use PrEP, whether high adherence could be sustained to achieve high effectiveness, and how to integrate these strategies into HIV-1 prevention programmes. Delivering these strategies in order to achieve population-level benefits is the next step, balancing costs, optimism, and realistic appreciation of the challenges ahead.

\section{PL05.2 NEW TECHNOLOGIES IN STI DIAGNOSIS AND CONTROL: PROMISING FUTURE}

doi:10.1136/sextrans-2013-051184.0013

C A Gaydos. Johns Hopkins University, Baltimore, MD, United States

Background Over 350 million (M) prevalent cases of curable STIs are estimated worldwide: $100 \mathrm{M}$ chlamydia (CT), 36M gonorrhoea
(NG), $187 \mathrm{M}$ trichomonas, and $36 \mathrm{M}$ cases of syphilis. $34 \mathrm{M}$ HIV infections are estimated worldwide. Human papillomavirus infections (HPV) and herpes viruses (HSV) are highly prevalent, yet are not often diagnosed. Technology has led to sensitive nucleic acid amplification tests (NAATs), allowing use of non-invasive specimens, like vaginal swabs and urine. NAATs are widely available, and novel approaches include point-of-care (POC) tests, internet availability, and self-testing. New serological tests have advanced diagnoses of HIV, HPV, and HSV. Yet, challenges to diagnosing STIs continue.

Methods To discern where we are heading in the field of HIV/STI diagnostics research, new assays and new approaches to enhance care were reviewed.

Results There are commercial NAATs for CT/NG by 4-5 companies. Some are available on expensive robotic platforms, limiting their use in resource-constrained settings. Additionally, a new CT/ NG NAAT that can return results in 90 minutes is available. Other rapid NAAT and hybridization POC assays are in the pipeline.

There are two NAATs and a POC test for trichomonas. POC syphilis serology tests have proliferated, which is important to syphilis elimination. Several platforms offer NAAT tests for HPV and there is a NAAT for HSV ulcers. Rapid HIV POC and $4^{\text {th }}$ generation tests have advanced earlier detection of HIV. A home oral-fluid HIV test can be purchased in the US. Patients perform their own HIV tests in Emergency Departments with excellent accuracy. Internet availability of select STI tests for home collection/outreach programmes offer novel approaches to increasing options offered to individuals at risk.

Conclusions Learning how to wisely and effectively use these tools can improve the detection of STIs and provide cost-effective ways to increase the number of patients being treated. 